\title{
IL Y A 100 ANS : LA SCHLITTE ET SES CHEMINS
}

La schlitte, peut-on lire dans un dictionnaire Larousse du début du siècle, "est un traîneau long, façonné pour recevoir du bois, et dont les patins se relèvent à l'avant en deux brancards arqués, entre lesquels se place le schlitteur pour diriger le traineau, et en modérer l'allure. L'emploi de la schlitte exige une voie spéciale, appelée chemin de schlitte».

La Revue des Eaux et Forêts consacrait, en 1891, à la schlitte, et aux chemins de schlitte, un intéressant article de fond ${ }^{(1)}$ dont nous extrayons les quelques pages que voici:

"Le schlittage consiste à charger les bois sur des traîneaux dont des hommes dirigent le glissement, le provoquant ou le modérant suivant les cas. Ces traîneaux, établis de façon à supporter des charges relativement fortes, sont fabriqués en hêtre bien sec par les schlitteurs eux-mêmes: les pièces en sont assemblèes au moyen de chevilles en bois. Ils permettent de transporter tous les produits: sciage, charpente et chauffage.

Les tronces de sciage sont débitées, les unes sur commande, à des longueurs variables; les autres, suivant l'ancien usage des Vosges, à 4 mètres. Ces dernières sont schlittees au bouc ou à la grande schlitte. Le bouc (figure 1) est assez court pour ne supporter qu'une extrémité de la tronce ${ }^{(2)}$; elle reçoit une très légère entaille, par laquelle elle prend appui sur la schlitte. On l'y fixe solidement par une chaîne que termine un petit coin fiché dans l'écorce ${ }^{(3)}$. La charge moyenne d'un bouc est d'environ 40 planches. Pour descendre les hétards ${ }^{(4)}$, dont un seul fait parfois de 60 à 80 planches, un même bouc est souvent dirigé par plusieurs hommes.

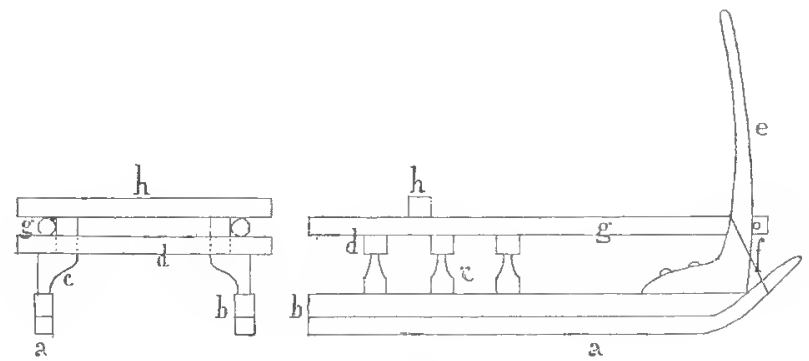

Figure 1.

(1) Jolly. - Les chemins de schlitte. - Revue des Eaux et Forêts, 1891, pp. 114-122.

(2) a. semelle, b. schlitton, c. pied, d. travers, e. corne, f. anneau en fil de fer, g. perche en sapin, h. double travers en sapin. La tigure 1 est à l'échelle de $1 / 20$; les figures 2,3 et 4 sont a l'échelle de $1 / 40$.

Une grande schlitte pèse environ 40 kilogrammes et revient à $18 \mathrm{~F}$; un bouc ou une schlitte à bois de corde pèsent 20 kilogrammes et reviennent à $8 \mathrm{~F}$.

(3) De cette façon, I'entaille et le coin sont egalement sans inconvenient; il n'en reste plus guere trace aprés que le sagard a apprëté la tronce a la hache. Ils ne pourraient d'alleurs abîmer que les dosseaux, marchandises de rebut qui ne se vendent souvent que comme chauffage.

(4) On donne ce nom aux plus grosses tronces, qui proviennent de la patte. 
On franchit ainsi des pentes variant de 50 à $20 \%$; le bouc ne soulevant qu'un bout de la tronce, tandis que l'autre traîne sur le sol, le frottement est considérable. Vers $20 \%$, il faut, pour faciliter le glissement, couvrir le sol de rameaux et de petites branches. Si la pente tombe au-dessous de ce chiffre et s'y maintient, le schlittage au bouc devient impossible. Alors on substitue au frottement de bois sur terre, le frottement moindre de bois sur bois. À cet effet, les tronces sont chargées entièrement sur une schlitte de longueur suffisante, la grande schlitte (figure 2), destinée à glisser sur des traverses espacées en moyenne de $0,60 \mathrm{~m}$ environ; ce sont les ravetons. La schlitte doit porter également sur tous; la pose en est donc faite avec soin: deux longues lattes, auxquelles on donne l'écartement des semelles de la schlitte, servent à régler suivant une succession de pentes régulières toutes les surfaces de frottement. Chaque raveton est solidement fixé en place par deux piquets (figure 3), qui le calent et qui maintiennent les schlittes dans la voie. D'autant plus rapprochés que la pente diminue davantage, les ravetons sont très espacés tant qu'elle demeure voisine de $20 \%$; dans ce cas, ils ne consistent qu'en de menues branches et ne donnent que très peu d'appui à la schlitte qui, dans leurs intervalles, frotte encore contre la terre. La pente devient-elle moins raide, on ravetonne en lattes de rebut, en sapin refendu, puis en rais de sapin; moins rapide encore, en quartiers de hêtre ${ }^{(5)}$; très faible, on attelle des bœufs aux schlittes. Est-elle assez prononcèe sur tout l'ensemble d'un trajet, les schlitteurs ont soin de munir leurs traîneaux de semelles tendres en bois vert; tandis que les semelles bien sèches, constamment graissèes, leur sont utiles dans les parcours à pentes réduites. Les alternatives de sécheresse et d'humidité, bien que moins sensibles en forêt qu'ailleurs, influent du reste beaucoup sur les conditions du glissement. Le beau temps l'accélère, en sorte qu'aux fortes pentes la poussée de la schlitte augmente; par contre, aux pentes faibles, l'effort de traction du schlitteur est moindre. La pluie produit naturellement les effets inverses.

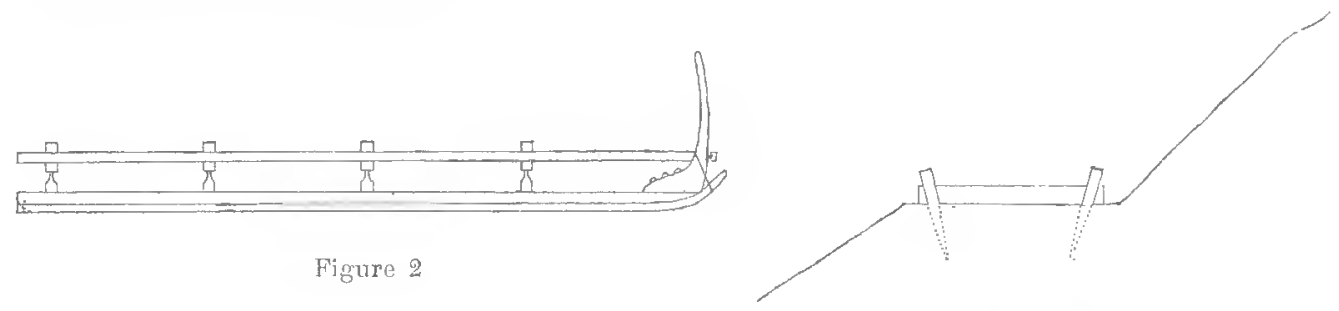

Figure 3

De même que le bouc, la grande schlitte reçoit en moyenne 40 planches; de même encore, quand, en raison de la charge ou des pentes, l'effort nécessaire devient excessif, il faut, pour descendre une seule schlitte, le concours de plusieurs hommes. Si la longueur des pièces dépasse 4 mètres, la grande schlitte est remplacée par un train de deux schlittes accouplées: la schlitte d'avant est un bouc ordinaire ; outre le schlitteur qui la dirige, un second ouvrier guide, au moyen d'une corde ou d'une perche, le mouvement de la chèvre, ou schlitte d'arrière. On peut enlever ainsi les sciages de toute longueur et les bois de charpente. Le chauffage enfin se transporte sur une schlitte munie de quatre montants (figure 4), entre lesquels se fait l'empilage. Elle sert sur toutes les pentes, mais tandis que sur les ravetons elle peut recevoir près de deux stères, souvent elle en porte à peine un demi sur les chemins de bouc.

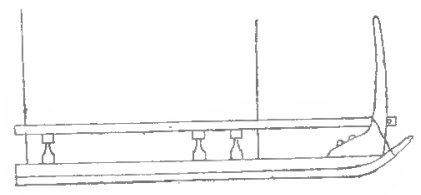

Figure 4.

(5) En gèneral, on ravetonne en rais de sapın entre 15 et $7 \%$ : les quartiers hëtre s'emploient quand, sur un certaın développement, la pente se maintient constamment au-dessous de $6 \%$. 


\section{II y a...}

En somme, le schlittage sur la terre nue, de 50 à $20 \%$, se distingue nettement du schlittage sur ravetons, au-dessous de $20 \%$. Celui-ci exige en général des chemins régulièrement établis aux frais du propriétaire. Le premier ne demande qu'un travail insignifiant que l'adjudicataire peut toujours prendre à son compte. Les schlitteurs tracent eux-mêmes les chemins de bouc; en quelques coups de fossoir ${ }^{(6)}$, ils pratiquent un passage suffisant, après avoir simplement écarté les roches. Au contraire, un chemin de schlitte à ravetons comporte un terrassement nivelé sur $1,20 \mathrm{~m}$ au moins de largeur. II nécessite donc une avance de fonds qu'un adjudicataire ne voudra presque jamais s'imposer, et que d'ailleurs une seule année d'exploitation ne pourrait pas toujours lui rembourser entièrement. Aussi les voituriers s'avancent-ils bien au-delà des chaussées empierrées, quelque misérables que soient, sur le grès vosgien, les conditions du charroi en terrain naturel ".

(6) houe vosgienne, à lame plate. 